\title{
Change in Serum Biomarkers in Patients with Osteoarthritis treated with Collagen Hydrolysate: Results of a Prospective Randomized Study
}

\author{
Klaus Flechsenhar ${ }^{1}$ and Timothy McAlindon ${ }^{2}$ \\ ${ }^{1}$ Sanofi-Aventis Deutschland GmbH, Frankfurt am Main, Germany
}

${ }^{2}$ Tufts Medical Center, Chief, Division of Rheumatology, Boston, USA

*Corresponding author: Klaus Flechsenhar, MD, Sanofi-Aventis Deutschland GmbH, Therapeutic Area Immunology, Cytokine Modulation, Industriepark Hoechst, Building H821, Room 525, D-65926 Frankfurt am Main Germany, Tel: +49 69305 17403; Fax: +49 69305 47223; E-mail: Klaus.Flechsenhar@sanofi.com

Received date: September 16, 2016; Accepted date: September 27, 2016; Published date: October 06, 2016

Copyright: (c) 2016 Flechsenhar K, et al. This is an open-access article distributed under the terms of the Creative Commons Attribution License, which permits unrestricted use, distribution, and reproduction in any medium, provided the original author and source are credited.

Keywords: Knee osteoarthritis; Nutritional supplement; Interventional trial; Imaging of cartilage; Serum chemical biomarkers

\section{Introduction}

Collagen hydrolysate is a nutritional supplement that is used for the treatment of patients with osteoarthritis. Collagen hydrolysate chemically speaking - is a mixture of proteins, namely collagen peptides, that are manufactured on the basis of skin and bones from cattle, pigs or fish on an industrial scale. Prospective, randomized, placebo-controlled trials demonstrated that the oral ingestion of 10 grams of collagen hydrolysate per day for the duration of three months decreases pain and improves function of joints in patients diagnosed with osteoarthritis [1-3].

As preclinical work had previously shown that collagen hydrolysate stimulates chondrocytes to synthesize extracellular cartilage matrix in a dose-dependent way, it appeared tempting to test whether collagen hydrolysate apart from improving signs and symptoms of osteoarthritis also exerts an impact on cartilage morphology, i.e. whether - despite being a nutritional supplement - collagen hydrolysate fulfills the properties of a disease-modifying drug, which is the capacity of changing both structure and symptoms. When researchers or sponsors of industry intend to generate scientific evidence that a certain drug or agent has a structure-modifying action, for regulatory purposes changes of joint space width as determined by $\mathrm{x}$-rays have to be demonstrated in prospective clinical trials. This particular endpoint in studies, i.e. changes of structure of the joint as determined by x-ray, is still part of the guidelines implemented by the Food and Drug Administration (FDA) [4] and the European Medicines Agency (EMA). However, during the past 20 years, a considerable body of evidence has been created, suggesting that the change of joint space width in the knee is more dependent on the shape of the meniscus [5] and that there is a poor correlation between cartilage morphology as visualized by magnetic resonance imaging and the changes of joint space width as determined by x-ray of the knee [6]. Thus, magnetic resonance imaging was used to test whether collagen hydrolysate exerts an impact on cartilage morphology which would also suggest structure-modifying properties. As method of magnetic resonance imaging, delayed gadolinium enhanced magnetic resonance imaging (DGMERIC) was specifically used for that particular study, as a very good correlation was shown between the T1-relaxation time and the density of proteoglycans in cartilage tissue [7]. In a prospective, randomized, placebo-controlled pilot trial, 30 patients with mild osteoarthritis of the knee (Kellgren-Lawrence I and II) were recruited and assigned to two groups, one group being treated with 10 grams of collagen hydrolysate per day and one group being treated with a placebo. All study participants had to ingest the nutritional supplement or the placebo for 48 weeks. DGEMRIC was performed at baseline, at 24 weeks and at 48 weeks. The primary endpoint of the study was the change of the T1-relaxation time from baseline in the region of interest at 24 weeks. The clinical trial was conducted under an FDA investigational new drug registration (IND \#74249) and registered on the ClinicalTrials.gov website (identifier NCT00536302) [7].

\section{Study Results}

It was interesting to see that at 24 weeks the T1-relaxation time (which is also called the DGEMRIC score) was higher in the treatment in comparison to the placebo group. With a Students two-tailed t-test comparison of change between the two groups, this difference was also statistically significant with a $\mathrm{p}=0.03$ in the medial tibia and a $\mathrm{p}=0.02$ in the lateral tibia. At 48 weeks, the DGEMRIC score in the treatment group was still superior to that in the placebo group, however the statistical significance was lost with a $\mathrm{p}=0.08$ and a $\mathrm{p}=0.07$ respectively. Symptoms were recorded in that trial, but no difference between groups was noted due to the small sample size.

When study participants - in the same study - presented for the baseline and the follow-up visits, blood was drawn and stored at $-80^{\circ} \mathrm{C}$ in a freezer. In those blood samples, we decided to measure two chemical biomarkers, namely PIIANP and CS846. PIIANP is a procollagen propeptide which is cleaved off from the collagen-type IIprecursor molecule and is most reflective of collagen synthesis. So, it can be regarded as an anabolic marker. CS846 (aggrecan chondroitin sulfate 846 epitope) is generated when the collagen and proteoglycan matrix is disrupted in cartilage. Thus, CS846 can be considered as a catabolic marker for cartilage matrix.

When we measured the changes of the concentration of the two biomarkers in the study participants, it was also interesting to see how those changes of serum biomarkers correlate with the changes of the DGEMRIC scores that were observed in the same study. As statistical method, a pairwise comparison of change in concentration and concentrations between groups was performed with Wilcoxon-tests.

As a matter of fact, at week 24 the PIIANP concentration increased in the treatment group and remained stable at week 48 . The change of the PIIANP concentration from baseline until week 24 between treatment and placebo group was statistically significant with a $\mathrm{p}=0.001$, while statistical significance disappeared at week $48(\mathrm{p}=0.7)$. The CS846 concentration increased at week 24 and 48 in the placebo group and statistical significance $(\mathrm{p}=0.045)$ was even noted between treatment and placebo group at week 24 . Thus, the data generated with the determination of biomarkers confirm the changes observed in the imaging scans [8]. 
Citation: Flechsenhar K, McAlindon T (2016) Change in Serum Biomarkers in Patients with Osteoarthritis treated with Collagen Hydrolysate: Results of a Prospective Randomized Study. J Arthritis 5: 219. doi:10.4172/2167-7921.1000219

Page 2 of 2

\section{Conclusion}

We are fully aware that our study comprises a small sample size, however, we believe that it may be seen as providing additional information to the epidemiological study of the Osteoarthritis Initiative. In that study, 4794 patients with osteoarthritis of the knee or risk factors for developing osteoarthritis of the knee are included and followed up annually for eight years. The purpose of that study is to correlate symptoms, imaging data and serum biomarkers in patients with osteoarthritis and to eventually be able to stratify patients according to risk factors and to identify markers that are predictive for progression of disease. The Osteoarthritis Initiative must be seen as an epidemiological study, whereas the collagen hydrolysate study mentioned above is an interventional study with positive findings when treatment and placebo groups were compared.

So, in our opinion, it would make sense to perform an identical collagen hydrolysate interventional study with a larger sample size $(n=200)$ in order to more robustly correlate symptoms, imaging data and serum biomarker concentrations. Such an interventional study, combining those three features, would contribute considerable knowledge to the field of osteoarthritis.

\section{References}

1. Moskowitz RW (2000) Role of collagen hydrolysate in bone and joint disease. Semin Arthritis Rheum 30: 87-99.
2. Jiang JX, Yu S, Huang QR, Zhang XL, Zhang CQ, et al. (2014) Collagen peptides improve knee osteoarthritis in elderly women. A 6-month randomized, double-blind, placebo-controlled study. Agro Food Industry Hi Tech 25: 19-23.

3. Kumar S, Sugihara F, Suzuki K, Inoue N, Venkateswarathirukumara $S$ (2015) A double-blind, placebo-controlled, randomised, clinical study on the effectiveness of collagen peptide on osteoarthritis. J Sci Food Agric 95: 702-707.

4. Food and Drug Administration (1999) Guidance for Industry on Clinical Development Programs for Drugs, Devices and Biological Products Intended for the Treatment of Osteoarthritis (OA).

5. Adams JG, McAlindon T, Dimasi M, Carey J, Eustace S (1999) Contribution of meniscal extrusion and cartilage loss to joint space narrowing in osteoarthritis. Clin Radiol 54: 502-506.

6. Wirth W, Duryea J, Le Graverand MPH, John MR, et al. (2013)Direct comparison of fixed flexion radiography and MRI in knee osteoarthritis: responsiveness data from the Osteoarthritis Initiative. Osteoarthritis Cartilage 21: 117-125.

7. McAlindon TE, Nuite M, Krishnan N, Ruthazer R, Price LL, et al. (2011) Change in knee osteoarthritis cartilage detected by delayed gadolinium enhanced magnetic resonance imaging following treatment with collagen hydrolysate: a pilot randomized controlled trial. Osteoarthritis Cartilage 19: 399-405.

8. McAlindon T, Bartnik E, Ried JS, Teichert L, Herrmann M, et al. (2010) Determination of serum biomarkers in patients with osteoarthritis: a previous interventional imaging study revisited. J Biomed Res. 